\title{
Blue intensity matters for cell cycle profiling in fluorescence DAPI-stained images
}

\author{
Anabela Ferro $^{1,2}$, Tânia Mestre ${ }^{3}$, Patrícia Carneiro ${ }^{1,2}$, Ivan Sahumbaiev ${ }^{3}$, Raquel Seruca ${ }^{1,2,4}$ and João M Sanches ${ }^{3}$
}

In the past decades, there has been an amazing progress in the understanding of the molecular mechanisms of the cell cycle. This has been possible largely due to a better conceptualization of the cycle itself, but also as a consequence of technological advances. Herein, we propose a new fluorescence image-based framework targeted at the identification and segmentation of stained nuclei with the purpose to determine DNA content in distinct cell cycle stages. The method is based on discriminative features, such as total intensity and area, retrieved from in situ stained nuclei by fluorescence microscopy, allowing the determination of the cell cycle phase of both single and sub-population of cells. The analysis framework was built on a modified k-means clustering strategy and refined with a Gaussian mixture model classifier, which enabled the definition of highly accurate classification clusters corresponding to G1, S and G2 phases. Using the information retrieved from area and fluorescence total intensity, the modified k-means $(k=3)$ cluster imaging framework classified $64.7 \%$ of the imaged nuclei, as being at G1 phase, $12.0 \%$ at G2 phase and $23.2 \%$ at $\mathrm{S}$ phase. Performance of the imaging framework was ascertained with normal murine mammary gland cells constitutively expressing the Fucci2 technology, exhibiting an overall sensitivity of $94.0 \%$. Further, the results indicate that the imaging framework has a robust capacity to both identify a given DAPI-stained nucleus to its correct cell cycle phase, as well as to determine, with very high probability, true negatives. Importantly, this novel imaging approach is a non-disruptive method that allows an integrative and simultaneous quantitative analysis of molecular and morphological parameters, thus awarding the possibility of cell cycle profiling in cytological and histological samples.

Laboratory Investigation (2017) 97, 615-625; doi:10.1038/labinvest.2017.13; published online 6 March 2017

\section{INTRODUCTION}

The cell cycle is a timely coordinated series of cellular events, aiming at the correct DNA replication and subsequent generation of two new, genetically identical daughter cells through cell division. It is a tightly regulated process, fundamentally ensuring the precise duplication and accurate segregation of the genomic and cytoplasmic content of cells in each cell cycle division. ${ }^{1}$ Under normal conditions, the eukaryotic cells in the resting/quiescent state (G0 phase) are diploid and have two sets of chromosomes $(2 \mathrm{~N})$. Upon reentering the cell cycle, diploid cells progress from G1 phase to an intermediate synthesis phase ( $\mathrm{S}$ phase) during which they initiate the doubling of DNA, protein and cytoplasmic content. At the end of this phase, cells are at G2 phase and become tetraploid $(4 \mathrm{~N})$. At this point, cells are ready to split and generate two new G1-diploid daughter cells, which ultimately occur after mitosis (M phase). Newly born cells may proceed to a new cell cycle or re-enter a resting state as quiescent cells. ${ }^{2,3}$ This ordered, unidirectional and wellestablished pattern of variation of DNA content is highly sensitive to intra- and extracellular signals and its fidelity is fundamental for cellular and tissue homeostasis. ${ }^{4}$

Several human pathologies, namely cancer, exhibit deregulation of the cell cycle. In fact, cancer cells frequently display cell cycle abnormalities and accumulate mutations, leading to unscheduled proliferation, genomic instability and aneuploidy. ${ }^{4}$ Hence, determination of cell ploidy status, S phase calculation and cell cycle dynamics have been explored as diagnostic tools relevant for therapeutic surveillance. ${ }^{5}$ In fact, cancer therapy response depends, in most cases, on the cell cycle phases. Although most chemotherapy drugs target broadly the active dividing cells, sparing cells at G0 state, as is the case of cisplatin, ${ }^{6}$ other treatments are more effective on a specific cell cycle phase; this is the case of methotrexate-based

${ }^{1}$ i3S - Instituto de Investigação e Inovação em Saúde, Epithelial Interactions in Cancer (EpIC) Group, Universidade do Porto, Porto, Portugal; ${ }^{2}$ IPATIMUP, Institute of Molecular Pathology and Immunology, University of Porto, Porto, Portugal; ${ }^{3}$ Institute for Systems and Robotics (ISR/IST), LARSyS, Bioengineering Department, Instituto Superior Técnico, Universidade de Lisboa, Lisboa, Portugal and ${ }^{4}$ Departamento de Patologia e Oncologia, Faculdade de Medicina da Universidade do Porto, Porto, Portugal Correspondence: Dr R Seruca, MD, PhD, Epithelial Interactions in Cancer (EPIC), i3S-IPATIMUP, Rua Alfredo Allen, 208, Porto 4200-135, Portugal.

E-mail: rseruca@ipatimup.pt

Received 13 October 2016; revised 23 December 2016; accepted 25 December 2016 
chemotherapy, which targets cells at $S$ phase, ${ }^{6}$ whereas radiation therapy is particularly effective on cells at G2 phase or undergoing cell division ( $\mathrm{M}$ phase). ${ }^{7}$ As such, the definition of the cell cycle momentum is critical to define the physiological status of individual or sub-populations of cancer cells, thus allowing adequate monitoring of therapy response.

In the last decades, an unprecedented technical development of cell biology methods has been witnessed. DNA content analysis progressed from highly laborious and timeconsuming methods to faster and quantitative state-of-the-art technologies. Moreover, the accessibility to DNA fluorochromes that bind stoichiometrically to DNA (eg, DAPI) allows the direct quantitative estimation of the DNA content in a biological sample. This principle has been largely explored in methods such as flow cytometry, image analysis and laser scanning cytometry. ${ }^{8}$ Among those, flow cytometry is considered the gold standard method for DNA content assessment and is the most disseminated method for singlecell interrogation. Despite its feasibility, reproducibility and affordability in multiparametric analyses, one of the major drawbacks of flow cytometry is exactly its basic principle: the fluidic stream. ${ }^{9}$ The crucial need for sample disaggregation leads to sample loss at post-analysis and the abrogation of the natural architecture of samples, which is critical when analyzing rare (on occasion, small) biological samples and for morphological-based analyses of adherent cell cultures and/or whole tissues. ${ }^{9}$ Therefore, imaging analyses software constitute a fast, accurate and cost-effective solution, as they allow the preservation of the natural architecture of cells. In fact, the application of digital technology to slide-format (for fixed samples) or plate-format (for live-cell imaging) enables quantitative determination of DNA content, as well as multiparametric analyses encompassing morphological, cellular and sub-cellular features of single cells.

In this study, we propose a new imaging analysis framework for the processing and quantification of fluorescence biological images by discretizing the cell cycle phase of cells from unsynchronized 2D cell cultures. The imaging framework tool relies on DAPI-binding intrinsic characteristics, which allow extraction of the nuclear features 'area' and 'total intensity' of DAPI-stained nuclei. Herein, we show that this new imaging framework is able to determine, with high accuracy, the cell cycle phase of individual cells and generate cell cycle profiles from cell populations based only on digital fluorescence images.

\section{MATERIALS AND METHODS Cell Culture}

NMuMG-Fucci2 cells (RCB2868, RIKEN Cell Bank, Japan) are normal murine mammary gland cells constitutively expressing the Fucci2 system: mCherry-hCdt1 (human Cdt1, 30/120 protein fragment) and mVenus-hGem (Geminin, $1 / 110$ protein portion), two chimeric proteins that accumulate, reciprocally, in the nucleus of transfected cells during the cell cycle. ${ }^{10,11}$ Fucci2 cells present cell cyclespecific fluorescence properties and function as G1 and S/G2/ M markers: nuclei of G1 phase cells are red, whereas nuclei of S/G2/M phase cells are green; cells transitioning from G1-to-S phase co-express both chimeras and exhibit a yellow nucleus. Cells were grown on top of glass coverslips in six-well plates with Dulbecco's modified Eagle's medium supplemented with $10 \%$ fetal bovine serum, 1\% penicillin/streptomycin and $10 \mu \mathrm{g} / \mathrm{ml}$ insulin in a humidified incubator at $37^{\circ} \mathrm{C}$ with $5 \%$ $\mathrm{CO}_{2}$. Cells cultures were kept at low passages and were grown up to $70-80 \%$ confluency.

\section{Cell Fixation and Staining}

Cells were washed with PBS $(\mathrm{NaCl} 137 \mathrm{mM}, \mathrm{KCl} 2.7 \mathrm{mM}$, $\mathrm{Na}_{2} \mathrm{HPO}_{4} 10 \mathrm{mM}, \mathrm{KH}_{2} \mathrm{PO}_{4} 7.4 \mathrm{mM}, \mathrm{pH}$ 7.4) and then fixed with $4 \%$ formaldehyde/PBS (freshly made) for $15 \mathrm{~min}$. Cells were then quenched for 10 min with $10 \mathrm{mM} \mathrm{NH}_{4} \mathrm{Cl}$ (PBS) and permeabilized for $10 \mathrm{~min}$ with $0.1 \%$ Triton X-100 (PBS). Nuclei were stained with $1 \mu \mathrm{g} / \mathrm{ml}$ DAPI (PBS) for $3 \mathrm{~min}$ and coverslips were then mounted on slides using plain Vectashield antifade mounting medium. All procedures were performed in the dark and at room temperature, unless stated otherwise. Mounted slides were kept overnight at $4{ }^{\circ} \mathrm{C}$, in the dark, before image acquisition.

\section{Fluorescence Imaging}

Fluorescence images were captured with a computer-assisted Zeiss Apotome Axiovert 200M Imager.Z1 upright microscope, equipped with a 40X/1.3 oil DICII(UV)VIS-IR objective lens (Carl Zeiss, Thornwood, NY, USA) with fixed illumination. DAPI images were collected with a G365 excitation filter, a FT395 dichroic mirror and a BP445/50 emission filter. These images were acquired in several planes along the $z$-axis $(60 \mathrm{Zs})$ while maintaining the acquisition settings: maximum pixel intensity at $50 \%$ and exposures of 5 ms. Images were captured with an Axiocam HRm camera and controlled with the Zeiss Axion Vision 4.8.1 software. Each DAPI image was then deconvoluted into a maximum intensity z projection of the 60 image planes centered at the focus plane, comprising a $16 \mu \mathrm{gm}$ in-depth nuclei imaging information retrieval. Fucci2-expressing cells were imaged with a BP450-490 excitation filter, a FT510 dichroic mirror, and a BP515-565 emission filter for mVenus/hGem, and a BP565/30 excitation filter, FT585 dichroic mirror, and a BP620/60 emission filter for mCherry/hCdt1. Single plane images $(1 Z)$ were obtained and used for the assessment of cell cycle stage of individual cells based on Fucci2-reporter's expression; the analysis was carried out with ImageJ software. ${ }^{12}$ This image set comprises the gold standard data set used for validation purposes.

\section{Pre-Processing Pipeline}

A pre-processing pipeline was applied to all the $2 \mathrm{D}$ in situ images in order to remove noise, increase contrast and adjust the dynamic range of image intensities, as described by 
Mestre et al (Figure 1b2). ${ }^{13}$ The application of these intensity conditioning procedures are crucial for the method proficiency, as both nuclei segmentation and cell cycle phasing depend on two key discriminative features of the stained nucleus, 'area' and 'total intensity'.

\section{Nuclei Segmentation and Feature Extraction}

Initially, a Gaussian $(\operatorname{sig}=2)$ smoothing filter was applied to DAPI images, where a $2 \mathrm{D}$ convolution operator was used to remove noise and blur the images. ${ }^{14}$ Noise removal and image intensity flattening enabled and improved the nuclei mask estimation by the generation of convex and path connected binary masks, which ultimately heightens the accuracy of the segmentation step. Next, sharpening of the resulting low-pass images was performed by using a high pass filter in order to enhance the transitions between nucleus/foreground and background regions. The intensity of images was then enhanced in order to reinforce the histograms bi-modality and proceed to the binarization stage, leading to nucleus final segmentation. The limiting function is $f\left(I_{i j}\right)=\left\{\begin{array}{cr}0 & I_{i j} \leq 0.1 \\ I_{i j} & 0.1<I_{i j}<0.5 \\ 1 & I_{i j} \geq 0.1\end{array}\right.$

Further, the saturated image was binarized using the Otsu method, ${ }^{14}$ to produce the final image mask. Small black regions $\left(I_{i j}=0\right)$ surrounded by white pixels that could not be reached by filling-in the background and connected components (white regions) with less than $N=200$ pixels were removed. Finally, regularization of the objects borders was attained with a morphological open operation of erosion followed by dilation, using a flat disk-shaped structuring element with radius $\mathrm{R}=\mu 2-\sigma$, where $\mu$ and $\sigma$ correspond to the average and standard deviation of all nuclei within an image, respectively. Segmentation of each nucleus was performed by computing the contour of all connected components within an image with the Moore-Neighbor tracing algorithm, modified by Jacob's stopping criteria. ${ }^{14}$ Hence, each sorted list of contour control points obtained represent the nucleus individually, from which it is possible to compute the mask $b_{i j}(\mathrm{k})$ representing a nucleus, according to $b_{i j}(k)=\left\{\begin{array}{cc}1 & (i, j) \in k^{\text {th }} \text { cell } \\ 0 & \text { otherwise }\end{array}\right.$. This mask was used to compute the area of each stained nucleus. However, owing to residual image noise or nuclei proximity or overlap, inefficient nuclei segmentation may occur. In these cases, our analysis framework was designed to encompass the possibility of complementary manual correction, allowing the operator to solve these difficulties.

\section{Cell Cycle Staging Imaging Framework}

Cell cycle staging classifier was established based on two nuclear features obtained through nuclei staining with DAPI: area, $A_{k}$, and total intensity, $T I_{k}$. The area was computed from the nucleus binary masks, according to $A_{k}=\sum_{i j} b_{i j}(k)$, and total intensity was computed also from these masks and by applying an element-wise multiplication to the original image, without any filtering, $T I_{k}=\sum_{i j} b_{i j}(k) I_{i j}$ thus avoiding distortion of intensity. For this, we estimated the label vector, $\boldsymbol{c}=\left[c_{1}, c_{2}, \ldots c_{N}\right]^{T}$ where $c_{k} \in\left\{G_{1}, S, G_{2}\right\}$ and $N$ is the number of nuclei in the image. The core of the method is an unsupervised multi-label classifier, where no prior knowledge regarding shape and absolute location of the clusters were assumed. However, two important biochemical concepts were introduced: (i) the DNA content of G2 nuclei is twice the $\mathrm{G}_{1}$ nuclei, ${ }^{2}$ and (ii) the fluorescence intensity derived from DAPI-stained nuclei is stoichiometrically proportional to the DNA present in each nucleus. ${ }^{15}$ Thus, the classification approach engaged into a two-stage classifier. The first stage comprised a modified version of the $k$-means classical unsupervised method, where the information concerning the expected DNA amount in $\mathrm{G}_{1}$ and $\mathrm{G}_{2}$ cell cycle phases was incorporated. Considering this, the centroids definition, $\mu$, for $\mathrm{G}_{1}$ and $\mathrm{G}_{2}$ clusters in the feature space are correlated as following, $\mu_{\mathrm{G} 2}=2 \mu_{\mathrm{G} 1} \quad$ (Supplementary Figure $1 \mathrm{~S}$ ). The classifications obtained in the first stage were used to initialize a Gaussian Mixture Model (GMM) classifier ${ }^{16}$ that tuned the final shape of the clusters. The clusters, in this second stage of the classification procedure, are described by multivariate normal functions, $N\left(\mu_{k}, \Sigma_{k}\right)$ where $\mu_{k}$ is the centroid of the cluster and $\Sigma_{k}=\alpha_{k} D_{k} A_{k} D_{k}^{\mathrm{T}}$ is the covariance matrix that defines the cluster shape; $D_{k}$ is an orthogonal matrix and $A_{k}$ is a diagonal matrix containing the eigenvalues of $\Sigma_{\mathrm{k}}$, whereas $\alpha_{\mathrm{k}}$ is scalar. The $\mathrm{D}_{\mathrm{k}}$ matrix defines the orientation of the ellipsoid of the cluster whereas $A_{k}$ defines its shape and $\alpha_{\mathrm{k}}$ its volume. The estimation of the cluster parameters, $\mu_{k}, \Sigma_{k}$, under the GMM is performed through the ExpectationMaximization (EM) method. ${ }^{16}$ In this imaging framework, the indicator (binary) variables, $Z_{i k}$ equal ' 1 ' when the $i^{\text {th }}$ nucleus is classified in $k^{\text {th }}$ and are estimated in a two-step iterative procedure. The classifications were obtained from $Z_{i k}$ through $c_{i}=\sum_{k=1}^{3} \mathrm{kz}_{i k}$. In the first step of the EM algorithm, latent membership weights are estimated to be used in the second step where the likelihood function is maximized. Both steps alternate until convergence is achieved. The covariance matrix used in the ensuing iteration, $\Sigma_{k}^{n+1}$, derived from the preceding estimation, $\Sigma_{k}^{n}$, as the following $\Sigma_{k}^{n+1}=\Sigma_{k}^{n}+A_{k}^{n} W_{k}^{n}$, where $W_{k}^{n}$ is the current relative amount of data in $k^{\text {th }}$ cluster. This incremental estimation of the covariance matrix aims at dealing with the ill-conditioned nature of the covariance matrix especially when the number of objects within the cluster is small. The initialization of the EM algorithm, $c^{\circ}$, was obtained from the classifications obtained with the modified $k$-means algorithm described previously. To circumvent the ill-conditioning of matrix $\Sigma_{k}$ and thus, the fluctuations of the iterative EM algorithm, because of small sizes of the clusters, for example, $N_{k}=1$, we used a spectral decomposition of $\Sigma_{k}$ in its inversion during the M-step of the EM algorithm. ${ }^{17}$ 


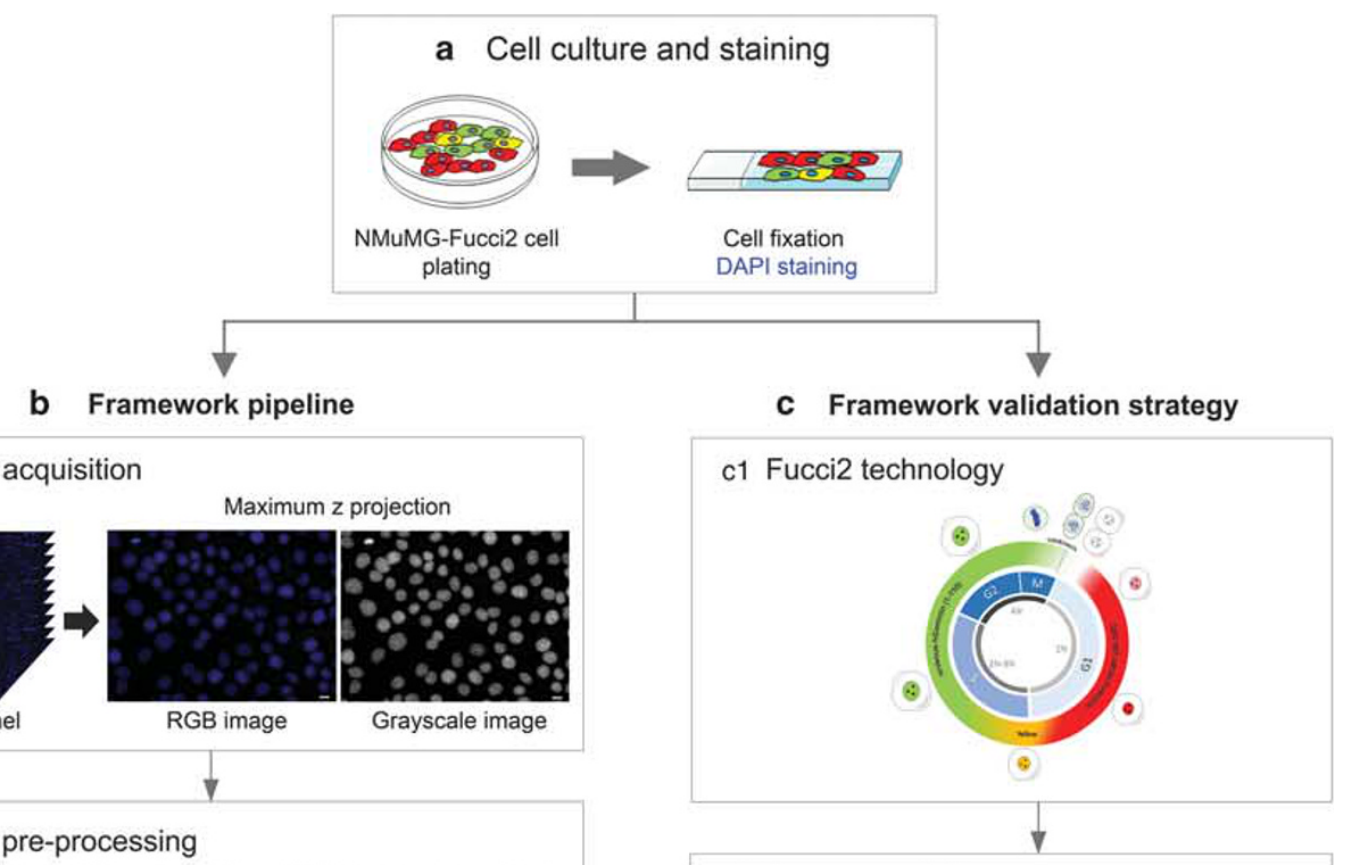

b2 Image pre-processing

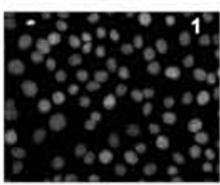

Denoising

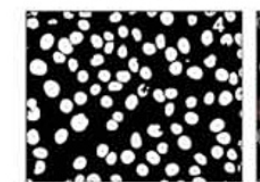

Otsu method output Nuclei segmentation

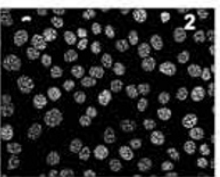

Contraste increase Intensity adjustment
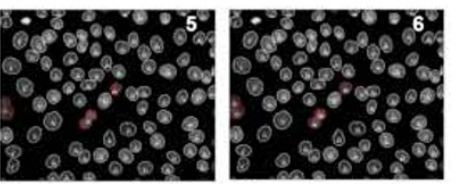

Manual correction

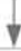

b3 Features extraction and cell cycle phasing outputs
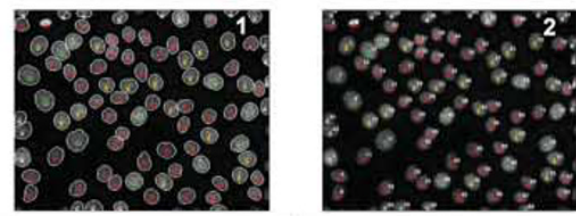

$\checkmark$

b4 Graphic representation of classification output
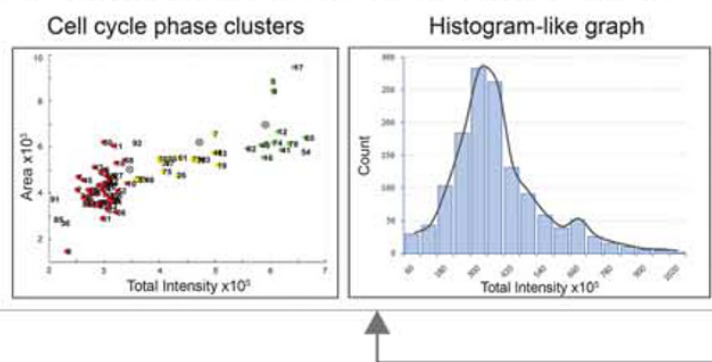

c2 Image acquisition
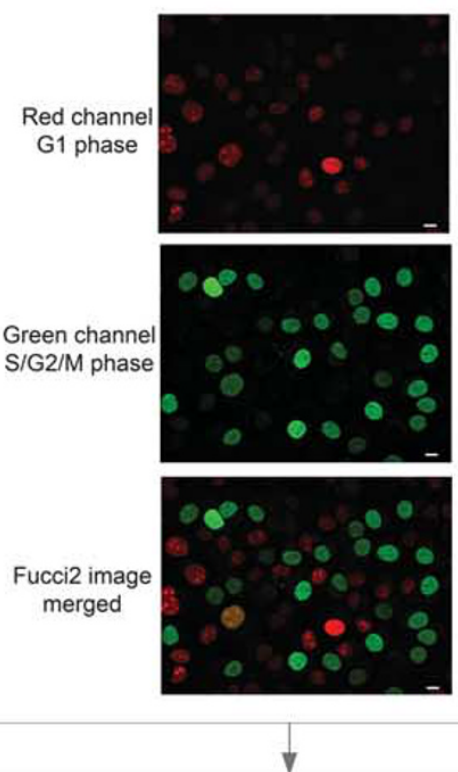

c3 Fucci2-based cell cycle classification

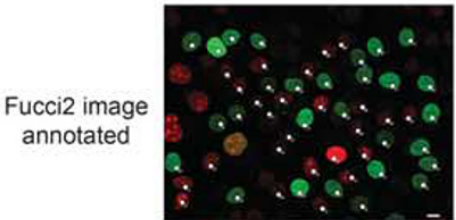

Validation of framework cell cycle profilling 


\section{Statistical Analysis}

Validation of the analysis framework against the gold standard data set, the Fucci2-based classifications, was performed by assessing the intrinsic characteristics of the imaging framework: sensitivity and specificity. The precision of sensitivity and specificity was estimated with the confidence intervals for a $95 \%$ confidence level. ${ }^{18,19}$ Statistical analyses were performed using an online software package (www. medcalc.org/calc/diagnostic_test). Further, the performance measures of the imaging framework to cell cycle stage $2 \mathrm{D}$ fluorescence images was ascertained with positive (PPV) and negative predicting values (NPV), that is, its value for predicting or excluding a cell cycle phase classification, respectively. ${ }^{18,20}$

\section{RESULTS}

\section{Imaging Framework for Cell Cycle Staging}

We developed an imaging framework to perform cell cycle analysis and classification based on fluorescence images of DAPI-stained nuclei obtained from routinely maintained 2D cell cultures. This framework relies on the fact that DAPI binds stoichiometrically to DNA, enabling the detection of DNA content variation occurring throughout the cell cycle progression. For this, denoising and nuclei segmentation was performed in each fluorescence image by applying the Otsu method and the Moore-Neighbor tracing algorithm, modified by Jacob's stopping criteria (Figure 1, Materials and methods section). ${ }^{14}$ Upon extraction of the features 'area' and 'total intensity' of DAPIstained nuclei, cell cycle classification was performed through a $k$-means clustering approach, enabling the classification of the imaged cells into discrete cell cycle subgroups or clusters (Figure $1 \mathrm{~b} 3$ and b4). Validation of the framework was performed using Fucci2-assisted cell cycle classification data set of the same NMuMG cells (Figure 1c1). ${ }^{10,21}$

\section{Imaging Processing and Features Extraction}

To establish an image-based cell cycle classification method of single cells, two nuclear features, 'area' and 'total intensity' were first digitally extracted from segmented DAPI-stained nuclei of 2D fluorescence images of NMuMG-Fucci2 cells. DAPI fluorescence staining was used to calculate the amount of DNA in a nucleus and, subsequently, infer its cell cycle status, through a $k$-means clustering approach. The area of each nucleus was determined as (1) Area $=\sum N_{P R}$. Total intensity of DAPI-staining yields, proportionally, the relative amount of DNA in each nucleus was calculated as (2) Total intensity $=\sum_{n=1}^{N} \operatorname{intensity}(n)$, in which $N$ is the total number of pixels within the nucleus. The $k$-means clustering approach was applied to the nuclear features, 'area' versus 'total intensity' (Figure 1b4) in order to attain cell cycle subgroups discretization, each referring to a distinct cell cycle phase. Distinct $k$-means strategies were applied by testing different number of clusters $(k)$ and types of data standardization (non-normalized, $z$-score standardization and weighted uncorrected s.d. normalization), but the final $k=3$ clustering approach generated the best fitted and biologically sound clusters (Figure 1b3). The capacity of the imaging framework to correctly segment the DAPI-stained nuclei and accurately extract relevant features for DNA content assessment of single cells is strongly dependent on sample preparation and image acquisition procedures. Hence, the conditions for optimal DAPI staining (yielding robustness but avoiding signal saturation) and the fluorescence microscopy acquisitions (light source intensity, exposure time) were optimized and maintained constant for all image sets analyzed.

Cell cycle profiling of asynchronous DAPI-stained cells with the imaging framework generated images with the annotation of the cell cycle phase for individual nucleus (Figure 1b3) and yielded cell cycle profiles with discrete representation of G1, S and G2/M phases, represented as cell clusters (Figure 1b4). With this type of classification outputs, it was possible to associate each single-cell/nucleus in the clustering plot to its cell cycle status, without the need of disruptive methods. Moreover, our results were consistent with the typical distribution of cells in G1, S and G2 phases

Figure 1 Overview of the imaging framework pipeline. (a) NMuMG-Fucci2 cells were grown on high-resolution microscope-quality coverslips and were then fixed. Cell nuclei were stained with DAPI. (b1) imaging acquisition was performed with a wide-field fluorescence microscope. DAPI images were acquired in several planes along the $z$ axis, deconvoluted into a maximum intensity z projection and then converted into grayscale images. (b2) The processing pipeline was applied to the grayscale images as follows: image was denoised (1), then its contrast was increased (2) and the intensity adjusted (3); the image was computed with Otsu's algorithm (4) and then nuclei were segmented (5); manual correction of the segmentation was performed when necessary (6). (b3) Nuclei features such as 'area' and 'total intensity' were extracted from DAPI-segmented nuclei (b2, 6) and cell cycle classification outputs were generated (b3, 1 and 2); the cell cycle stage of nuclei at images borders was not ascertained; red, green and yellow colors in the classification outputs correspond to G1, G2/M and S phase cells, respectively, and were automatically assigned by the framework. (b4) Cell cycle cluster graphs were then calculated based on the 'area' and 'total intensity' features from (b3), and cells were clustered by the framework according to its predicted cell cycle phase; the red, green and yellow clustered dots correspond to G1, G2/M and S phase cells, respectively. Alternatively, a histogram-like graph depicting the cell cycle profile may be plotted from the generated Excel outputs. (c1) Validation of the imaging framework was performed by using a well-established cell cycle sensor technology (Fucci2), constitutively expressed by NMuMG cells. (c2) For this, single plane images of the red and green channels were captured from the same field-of-view of the blue (DAPI) channel described in (b1). (c3) Red and green images were combined, annotated and the cell cycle classification of each Fucci2-expressing cell was ascertained by visual inspection. Finally, the performance of the imaging framework was assessed by comparing the cell cycle classification profiles obtained with the framework with those from the Fucci2-derived assessment. Scale bar corresponds to $10 \mu \mathrm{m}$. 
described in the literature, fitting an histogram-like representation (Figure 1b4), supporting the relevance of the extracted features 'area' and 'total intensity' of DAPI-stained nuclei in the cell cycle phasing of digitized images.

\section{Imaging Framework Performance}

To validate the imaging framework, we applied it to fluorescence images obtained with unperturbed NMuMGFucci2 cell cultures counterstained with DAPI (Figure 1c1). This Fucci2 cell model is a well-established cell cycle sensor that allows an easy and colored visualization of the cell cycle phase at single-cell level. ${ }^{11,21}$ In particular: red-fluorescent nuclei expressed the mCherry-hCdt1chimeric protein and were classified at G1 phase; green fluorescent nuclei, expressed the mVenus-hGem fusion protein and were considered as replicating cells at S/G2/M phases (Figure 1c2); nuclei that exhibited a yellow coloration coexpressed both cell cycle reporters, and were considered as transitioning from (late) G1 phase to (early) S phase. Using these Fucci2 cells, we obtained a large data set of images acquired with DAPI channel (all nuclei) and with green and red channels, which are representative of Fucci2 cell cycle reporter's expression. DAPI channel images were subjected to the imaging framework for cell cycle phase classification, whereas the red/green channel imaged cells were independently classified into distinct cell cycle phases (gold standard) (Figure 1c3) and used for validation of the results obtained with the imaging framework.

We surveyed a panel of 13 data sets of images (corresponding to eight independent cell passages of NMuMG-Fucci2 cells), comprising a total of 142 images from DAPI, red and green channels, each with a resolution of $1388 \times 1040$ pixels. To maximize the information retrieved from each DAPIstained nucleus (fluorescence total intensity), we collected 60 $Z s$-stacks for each image. Each DAPI image was deconvoluted into a maximum intensity $\mathrm{Z}$ projection, corresponding to a total of $16 \mu \mathrm{m}$ in-depth nuclei imaging information retrieval. In total, we imaged 10805 DAPI-stained nuclei of Fucci2 cells, with an average of 72 nuclei per image. Nuclei at image borders were not considered for analysis, as the information retrieved from these objects would be incomplete; these events accounted for $14.7 \%(n=1583)$ of all nuclei imaged. From all nuclei imaged, we ascertained the cell cycle phase of $85.4 \%(n=9223)$. From these, the modified $k$-means $(k=3)$ cluster imaging framework classified $64.7 \%(n=5970)$ of nuclei as being at G1 phase, $12.0 \%(n=1111)$ as G2 phase nuclei, and assigned $23.2 \% \quad(n=2142)$ to the $S$ phase (Table 1). The gold standard data set (Fucci2-based classification) comprised $43.6 \%(n=2918)$ of Fucci2 cells as red cells (G1 phase), $24.7 \%(n=1652)$ as yellow cells, thus in transition from G1-to-S phase, and 31.6\% $(n=2115)$ as green cells (S/G2/M phase) (Table 1). The framework-assisted cell cycle classification was compared with the gold standard classification data set. Given that the imaging framework generated a classification for a higher number of cells than the
Table 1 Characteristics of the cell populations assessed by the imaging framework and derived from the Fucci2 cell model analysis. The latter was used for the imaging framework validation

$n(\%)$

\begin{tabular}{lc} 
Algorithm & 10805 \\
All DAPI-stained nuclei imaged & $1583(14.7)$ \\
Rejected $^{a}$ & $9223(85.4)$ \\
Classified & \\
Population distribution & $5970(64.7)$ \\
G1 & $2142(23.2)$ \\
S & $1111(12.0)$ \\
G2/M & 9223 \\
Fucci2-cell model & $2535(27.5)$ \\
All cells inspected & $6688(72.5)$ \\
Fucci2-negative cells (colorless) & \\
Fucci2-expressing cells & \\
Population distribution & $2115(31.6)$ \\
Red cells (G1) & $1652(24.7)$ \\
Yellow cells (G1/S) & \\
Green cells (S/G2/M) & \\
\hline
\end{tabular}

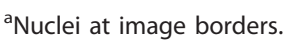

${ }^{b}$ Both colorless cells at the time of the analysis and abnormally big Fucci2expressing cells.

Fucci2 system ( $n=9223$ versus 6688 nuclei), only those cells classified by both methodologies were used for comparison and validation. This difference in cell numbers is due to intrinsic features of the Fucci2 system. Despite that NMuMGFucci2 cells are genetically engineered to stably express two cell cycle reporters, not all cell cycle phases are visible; this is the case of newly formed G1 cells, which are colorless thus contributing to a Fucci2-validation void.

The performance of the imaging framework was ascertained by calculating its sensitivity and specificity. As measures of the population proportions, the sensitivity and specificity may be characterized by confidence intervals calculated based on standard methods for proportions. ${ }^{22}$ The corresponding 95\% confidence intervals were calculated resorting to an online calculator (see Material and methods). ${ }^{18}$

So, how good is the imaging framework at cell cycle staging of DAPI-stained 2D fluorescence images? Ideally, an imaging method would be a powerful, discriminative tool, characterized both by high specificity (with low false positive rate, $\alpha$ ) and sensitivity (with low false negative rate, $\beta$ ). ${ }^{23}$ Regarding its ability to correctly classify the Fucci2-red cells as true G1 cells (Table 2), the imaging framework showed a sensitivity of 94.6\% (95\% CI: $93.7-95.3$ ) and a specificity of $93.3 \%$ (95\% CI: 
92.5-94.1) (Table 3). Moreover, the imaging framework showed a sensitivity of 90.3\% (95\% CI: 89.0-91.5) (Table 3), denoting a high capacity to assign correctly the Fucci2-green cells as true G2/M cells. Despite this high ability, we concluded that when the algorithm was analyzing DAPI images from green cells it showed less ability to identify them as true G2/M cells (Table 2, true positives); nevertheless, it was able to determine the true negatives with a specificity of $95.5 \%$, (95\% CI: 94.8-96.1) (Table 3), that is, to correctly identify, from all DAPI-stained nuclei, those that are not true G2/M cells (Table 2, true negatives). Validating the imaging framework with the Fucci2 cell cycle sensor allowed us to classify cells transitioning from G1-to-S phase, which exhibited a yellow color when visually inspected (Figure $1 \mathrm{cl}-\mathrm{c} 3$ ). This group of G1/S cells allowed the validation of both G1 and S phase cells as these yellow cells are simultaneously at late G1 phase and early S phase. ${ }^{10}$ Strikingly, our imaging framework exhibited a sensitivity of $97.1 \%$ (95\% CI: $86.1-97.9)$ and a specificity of 92.8\% (95\% CI: 92.0-93.5) (Table 3) to identify yellow cells as being true G1 or true S phase cells (Table 2).

Table 2 Cell cycle classification results from the imaging framework for the Fucci2-positive cells analyzed

\begin{tabular}{|c|c|c|c|c|c|c|}
\hline \multirow{2}{*}{$\begin{array}{l}\text { Cell } \\
\text { cycle } \\
\text { phase }\end{array}$} & \multicolumn{3}{|c|}{ No. } & \multicolumn{3}{|c|}{ No. } \\
\hline & $\begin{array}{c}\text { True } \\
\text { positive }^{a}\end{array}$ & $\begin{array}{c}\text { False } \\
\text { negative }^{b}\end{array}$ & Total & $\begin{array}{c}\text { True } \\
\text { negative }^{c}\end{array}$ & $\begin{array}{c}\text { False } \\
\text { positive }^{d}\end{array}$ & Tota \\
\hline G1 & 2745 & 158 & 2903 & 3488 & 250 & 3738 \\
\hline S & 1596 & 47 & 1643 & 4637 & 361 & 4998 \\
\hline $\mathrm{G} 2 / \mathrm{M}$ & 1892 & 203 & 2095 & 4341 & 205 & 4546 \\
\hline
\end{tabular}

${ }^{a}$ True positive indicates a cell that was of a given color and was classified accordingly by the framework.

${ }^{b}$ False negative indicates a cell that was of a given color and was classified otherwise by the framework.

'True negative indicates a cell that was of any other color and was classified accordingly by the framework.

${ }^{d}$ False positive indicates a cell that was of any other color and was classified otherwise by the framework.
To explore the applicability of the imaging framework for the classification of cell cycle stage based on nuclear staining, we determined its associated predictive values. ${ }^{24}$ Hence, the probability of the imaging framework to correctly classify a given cell cycle stage, when the gold standard states as such or otherwise were assessed by estimating its associated PPVs and NPVs, respectively. ${ }^{20,24}$ The measure of precision of the estimated ratios was assessed with their 95\% confidence intervals. ${ }^{18,20}$ The PPVs were consistently high for all cell cycle classifications: 91.6\% (95\% CI: 90.6-92.6) for G1 cells, 90.2\% (95\% CI: 88.8-91.5) for G2/M cells and 81.5\% (95\% CI: 79.7-83.2) for G1/S transitioning cells (Table 3). The ability of the imaging framework to exclude a phase classification (NPV) associated with each cell cycle phase were also consistently high: 95.7\% (95\% CI: 94.6-96.3) for G1 cells, 95.5\% (95\% CI: 94.9-96.1) for G2/M cells and 99.0\% (95\% CI: 94.9-96.1) for G1/S cells. ${ }^{18}$ These results indicate that the imaging framework has a robust capacity to both identify, with 95\% confidence, a given DAPI-stained nucleus to its correct cell cycle phase, (ie, true positives), as well as to determine, with very high probability, to which cell cycle phase a specific DAPI-stained nucleus does not belong (true negatives). This implies that the imaging framework reported very few false negatives in all cell cycle phases analyzed (Table 2). As predictive values are dependent on the cell cycle sub-phase proportion within the cell cycle, it is not surprising that the highest (91.6\%) and the lowest $(81.5 \%)$ estimated PPV are for G1 and S phase classification, as these are the most and least abundant cell cycle phases, respectively, in unperturbed cell cultures. Accordingly, the highest NPV $(99.0 \%)$ is associated with the $\mathrm{S}$ phase, the least abundant cell cycle phase.

The sensitivity (or power) of a test is the probability of correctly rejecting the null hypothesis when it is false, that is, the probability of not entailing in type II errors. ${ }^{23}$ Hence, the power of analysis is related by $1-\beta$ to the type II error rate $(\beta) .{ }^{18}$ In this regard, we concluded that this new imaging framework reported very few type II errors (false negatives) in all cell cycle phase classifications (Table 2), which translated into high power to classify the DAPI-stained nuclei to their

Table $\mathbf{3}$ Imaging framework classification characteristics, sensitivity and specificity, were calculated considering a 95\% confidence
level
\begin{tabular}{lccccc}
\hline Cell cycle phase & Sensitivity, \% (Cl) & Specificity, \% (Cl) & Power $\left(1-\beta^{\mathrm{a}}\right), \%$ & Positive predictive value, \% (Cl) & Negative predictive value, \% (Cl) \\
\hline G1 & $94.6(93.7-95.3)$ & $93.3(92.5-94.1)$ & 94.6 & $91.6(90.6-92.6)$ & $95.7(94.6-96.3)$ \\
S & $97.1(96.2-97.9)$ & $92.8(92.0-93.5)$ & 97.1 & $81.5(79.7-83.2)$ & $99.0(98.7-99.3)$ \\
G2/M & $90.3(89.0-91.5)$ & $95.5(94.8-96.1)$ & 90.3 & $90.2(88.8-91.5)$ & $95.5(94.9-96.1)$
\end{tabular}

Abbreviation: $\mathrm{Cl}$, confidence interval.

The performance of imaging framework was also assessed by calculating the associated positive and negative predictive values.

${ }^{a}$ False negative rate $(\beta)$ was calculated as (false negatives/(true positives + false negatives)). Statistical analyses were performed using an online software package (www.medcalc.org/calc/diagnostic_test). 
correct cell cycle phase: $94.6 \%$ for stained nuclei at G1 phase, $97.1 \%$ when assigning at G1/S phase cells and $90.3 \%$ for G2/M cells (Table 3 ).

\section{DISCUSSION}

Herein we describe a new imaging framework designed to classify the cell cycle stage of individual and cell sub-populations from in situ DAPI fluorescence images (Figure 1b1). The imaging framework is based on a processing pipeline comprising a denoising algorithm, contrast and intensity adjustments, Otsu's computation, nuclei segmentation and an optional manual correction tool (Figure 1b2). The framework generates final images depicting the segmented DAPI-stained nuclei derived from 2D images and pinpoints the cell cycle classification of each nucleus analyzed (Figure 1b3). In a semi- or fully automated manner, it is possible to segment DAPI-stained nuclei and generate, based only on the 'area' and 'total intensity', simple and accurate clusters and histogram-like graphs fitting the cell cycle profile of DAPIstained nuclei (Figure 1b4). It is a very simple, rapid and inexpensive imaging platform, thus easily standardized. Moreover, as there is no need of a disruptive method for sample preparation, it is possible to retrieve from the digitized cells, a plethora of quantitative data (eg, expression pattern of proteins or nucleic acid of interest and morphology-derived parameters). Furthermore, these data can be correlated with all or a specific cell cycle phase, under physiological conditions, awarding this new imaging framework analytical properties unattained by others. To validate the imaging framework, we used a well-established Fucci2 cellular model (NMuMG-Fucci2 cells), which allowed for the colored readout of the cell cycle phase of each cell digitized.

Overall, the imaging framework exhibited high classification accuracies across all cell cycle phases. With high sensitivity (and specificity) for all cell cycle phase, it revealed a high ability to recognize the correct cell cycle phase of DAPI-stained nuclei. The sensitivity or power analysis of binary testing (true/false classifications) represents the probability, in this case, of the imaging framework to classify correctly, with reasonable certainty, the cell cycle phase of DAPI-stained nuclei identified by Fucci2 technology accordingly. On the other hand, specificity represents the ability of the imaging framework to identify the cell cycle phase of stained nuclei, classified otherwise by the gold standard, without entailing in type I errors (false positives). The imaging framework showed to be a powerful tool to correctly identify the cell cycle phase of DAPI-stained nuclei, which means that there is a low probability associated with this new DAPI-based cell cycle method to entail in type II errors during cell cycle phasing, that is, in producing false negative results.

Based solely on DAPI staining, we were able to determine with the highest probability, the true G1 nuclei (PPV $=91.6 \%$ (95\% CI: 90.6-92.6)); one the other hand, the imaging framework showed less capacity to identify the $S$ phase nuclei
$(\mathrm{PPV}=81.5 \%(95 \%$ CI: 79.7-83.2)). It is conceivable that these results are related to the validation system chosen. The Fucci2 cell system enables a dichotomic classification: red cells are in G1 phase and green cells are in S/G2/M phase (Figure 1c1). Cells at $\mathrm{S}$ phase do not express an unequivocal coloration, which raises some difficulties in the validation of these DAPI-stained nuclei. Still, the imaging framework was able to assign the DAPI-stained nucleus as being at $S$ phase with an $81.5 \%$ likelihood. The imaging framework may be further refined, particularly in its S phase predictive capacity, by upgrading the complexity of the validation system with a second marker, which would potentiate the framework classification at this cell cycle phase. Overall, the framework showed an impressive accuracy to assign each DAPI-stained nucleus to its correct cell cycle phase. Currently, this new imaging framework is a robust, quantitative image-based cell cycle classifier, capable of accurate and biological sound cell cycle phase profiling of individual cells.

This imaging framework strongly depends on the correct identification and segmentation of nuclei, in order to accurately estimate the DNA content and profile the cell cycle stage. To cope with these difficulties, we introduced the possibility to manually correct the automatic segmentation (Figure 1b2). Although more time consuming, this possibility will be particularly relevant for future applications in the accurate segmentation of stained nuclei from tissue sections, or for the establishment of biological criteria defining the nuclei of interest within the 2D fluorescence images. This semi-automatism has been proven valid by Sanches et $a^{25}$ in the selection of biological sound cell-cell pairs to profile protein levels and distribution on in situ fluorescence images of heterogeneous cell populations expressing normal or mutant E-cadherin.

The information retrieved from the DAPI-stained nuclei was collected with multi-stacking planes. In this regard, we assured that the mitotic cells were also well captured in the in situ fluorescence images. This is important since during mitosis, cells detach from the cell lawn and round-up because of cell division constraints. ${ }^{26}$ As mitosis can be easily detected by visual inspection, their classification by the imaging framework was not our focus when designing this DAPIbased cell cycle classifier. But, in fact we observed that early stages of mitoses (ie, prophase and metaphase) are accurately included into the G2/M phase by the imaging framework (unpublished data). However, this does not hold true for later stages of mitosis (ie, anaphase and telophase). The late-stage mitotic nuclei were incorrectly identified as two independent, small-area objects leading to a false G1 classification. In this regard, we agree with others ${ }^{27}$ that these misclassifications lead to a smaller G2/M population ( $12.0 \%$ for our study) compared with flow cytometry classifications of unsynchronized cell cultures. ${ }^{21}$ Strikingly, the lowest sensitivity (or power) was associated with the classification of the G2/M population (90.3\% (CI: 89.0-91.5)). Therefore, for mitotic-related studies with this imaging framework it is 
recommended the usage of additional mitotic markers (eg, phospho-histone H3) to increase the sensitivity in the classification of the G2/M population.

Our imaging approach allowed the validation of the exact same individual cell nucleus used for cell cycle classification through DAPI-intensity and Fucci2-color readout. Our validation procedure was done preserving the natural architecture of NMuMG-Fucci2 adherent cells, unlike other studies, which used cell detachment and flow cytometry to evaluate cell cycle staging. ${ }^{27}$ Moreover, our imaging framework enables the operator to match each classified cell to its cell cycle phase and correlate it with other parametric measurements performed in the same field-of-view of a $2 \mathrm{D}$ fluorescence image.

\section{Comparing with Other Methods}

The visualization and profiling of the cell cycle is often dependent on antibody-based methods that detect cell cycle regulatory proteins specific of a cell cycle phase (eg, cyclin B1 for mitosis, cyclin D1 for G1 cells, etc.) or estimate the number of cycling cells (eg, using BrdU or EdU for S phase cells). ${ }^{3}$ Despite the high specificity and efficiency, an antibody-based strategy to profile the complexity of the eukaryotic cell cycle depends on the combination of multiple markers specific for each phase, highly impacting on the feasibility of the method. Moreover, the analysis of several fluorescence markers often lead to problems of fluorescence spectra overlap, even when highly discrete fluorescenceemitting moieties, like quantum dots, are chosen. ${ }^{28}$ Thus, the increased complexity of multiparametric analyses, allied with the imperative need of several fluorescence channels for the microscopy analysis, often renders these approaches technically challenging. These difficulties may be circumvented by cell synchronization protocols, empirically defined for each cell model, which are often used to perform correlative studies for specific cell cycle phases. Nevertheless, these are very restrictive cellular analyses and may introduce artifacts to the cell cycle analysis; cells are exposed to external stresses, thus hampering the analysis of unperturbed cellular systems. ${ }^{29}$ The possibility to use exogenously expressed cell cycle markers, as Fucci-assisted reporter systems (reviewed in 30) facilitated the quantitative analysis of cell cycle status, especially when studying complex systems as whole animals. ${ }^{31-33}$ Despite the fact that the development of these fluorescence cell cycle sensors came to revolutionize our ability to see the cell cycle, especially in live-cell imaging-based techniques, these genetically engineered systems (i) do not tackle the bleed-through problems inherent to conventional fluorochrome-dependent analyses, (ii) are time-consuming to establish and (iii) display some pitfalls in the identification of some cell cycle phases. ${ }^{30}$ Conversely, the image-based framework presented here depends only on cell nucleus staining to profile the cell cycle of unsynchronized 2D cell cultures. It is a straightforward and inexpensive alternative for cell cycle profiling and accurately classifies all cell cycle phases, including S phase cells, outperforming the Fucci-cell models. Although the results presented are based on DAPI staining, it is conceivable that the use of other cell-impermeant dyes (eg, DRAQ7, propidium iodide, FxCycle ${ }^{\mathrm{TM}}$ dyes, etc), will generate the same cell cycle profiling robustness and efficiency.

Other high-resolution imaging formulations for DNA content measurements have been reported as allowing cell cycle staging of single cells through the analysis of fluorescence intensity. ${ }^{27,29,34}$ Namely, Bruhn et al ${ }^{29}$ established a two-color method that enables the study of cell cycledependent cellular and sub-cellular processes. The elegant analysis pipeline of cell cycle profiling is based on DAPI staining but it also depends on an EdU click-labeling reaction to define the cycling cells at $S$ phase. Although EdU click-it reaction seemed the obvious choice to avoid antibody-based methods for S phase discrimination, it limits the method especially for those GFP-assisted protocols because of the copper-associated quenching of GFP fluorescence. On this regard, our imaging framework is simpler, broader and more cost effective. It surpassed the need of an additional $S$ phase marker for the cell cycle clustering task as it incorporated the prior biological knowledge that G2 cells have twice the DNA content of G1 cells and S phase cells intermingle with the latter. This principle assisted the maximization of the imaging framework classification performance and improved stability on cell cycle phasing. Moreover, it generated statistically robust and accurate definition of all cell cycle phases based solely on DAPI staining of 2D cell cultures. This narrows down the complexity of the analysis and costs as it depends on a reagent commonly found in laboratories. Recently, Roukos et $a^{27}$ also reported a cell cycle staging method devised on DAPI staining of cells. However, our new imaging framework is singular in that it has the possibility to (i) generate final images displaying cell cycle classification of individual cells among the analyzed in situ fluorescence cells, and (ii) allows manual inspection or correction of the segmented data, enabling the user some control over the biological relevance of the segmentation produced, and (iii) to profile the cell cycle status of the entire digitized cells population, but also the possibility to focus the analysis into a specific sub-population of cells at a specific cell cycle phase, and consequently, assess them individually.

\section{Considerations and Limitations of the Analysis Framework}

The proposed imaging framework depends on the accurate estimation of the DNA content of 2D fluorescence images. Hence, the correct segmentation of DAPI-stained nuclei is essential. Being a microscopy-based approach, parameters including sample preparation, homogeneous illumination of the sample, light source intensity and exposure times need to be determined empirically for each assay and maintained constant during image acquisition. These are decisive parameters for the generation of accurate data, either applying this imaging framework to low or high-throughput analyses. 
We have successfully used this protocol based on DAPIstained nuclei analysis to explore the impact of E-cadherin dysfunction upon the morphometric features of in vitro cell cultures. ${ }^{13}$ Applying the above-mentioned criteria, this imaging tool renders the possibility to complement the cell cycle staging with other fluorescence-based multiparametric analyses. This imaging framework can be adapted to different samples as long as the DNA staining protocols enable a quantitative assessment of the DNA based on fluorescence. Overall, the imaging framework allows quantitative and statistically robust analysis of cell cycle phase-dependent cellular properties under physiological and non-disruptive conditions in a large number of samples, namely molecular pathology. One of the most disseminated technology applied to molecular pathology, both at research and diagnostic settings, is the tissue microarray (TMA) technology. ${ }^{35}$ TMA is a fast, reproducible and quantitative technique, enabling the analysis of hundreds of patient tissues arrayed in a single microscope slide. Although it has been extensively applied to archived, paraffin-embedded samples through immunohistochemistry, TMA has been adapted to fluorescence-derived analysis, namely DAPI staining, which widened its analysis range and information retrieval. ${ }^{35}$ In this sense, the application of the imaging framework to TMA is highly feasible. Nevertheless, it should be noted that its application to histological analyses requires empirical optimization of sample processing protocols, in order to minimize tissue-related cumbersome associated with histological procedures. ${ }^{36}$ For instance, nuclei shrinkage is associated with paraffin-based protocols, which impacts on nuclei area and numerical density, as cells appeared closer to each other. ${ }^{37}$ Factors as cell clustering or overlapping, tissue heterogeneity and autofluorescence observed in fluorescence-stained histological samples may, in fact, lead to missegmentation of nuclei. ${ }^{38}$ These are, undoubtedly, relevant aspects to be consider when using this framework for histological analysis, as the intrinsic characteristics of this imaging framework derive from area and total intensity of DAPI-stained nuclei. Nevertheless, our segmentation algorithm has proven to be very efficient for nuclei or cell segmentations, ${ }^{13,25}$ and in fact its semiautomatism enables the operator to critically evaluate and even define nuclei segmentation in histological samples. To perform a comprehensive analysis of more complex samples resorting to this imaging framework, image acquisition procedures should follow the recommendations stated herein. For instance, imaging should be performed with a microscope capable of in-depth, z-stacking acquisition and the final images should represent a maximum projection of the $z$-stacking imaging in order maximize sample information. We envisage that image analyses of frozen tissue sections will required less optimizations as nucleus staining protocols based on fluorescence dyes are seemly and there are less morphological constraints when samples are frozen. The application of the imaging framework to molecular pathology, such as in cancer, will increase the analysis potential of DAPI-stained images given that it is possible to correlate cell cycle information with morphometric and expression patterning data, which may ultimately represent an important advantage in cell research and clinical practice. This knowledge will increase diagnostic accuracy, create novel biological-based therapies and set-up more precise clinical protocols for disease treatment and patient survey, namely in cancer.

Given the proven discrimination and classification power of the imaging framework we envisage that to assess the cell cycle stage of clinical-derived samples (eg, tumor samples), with high certainty ( $95 \%$ confidence level) and without the previous knowledge of the total cell number of the sample, we would have to analyze approximately 380 cells from the region of interest of the tumor sample in order to perform a representative analysis of the whole tumor. Sample size calculations were made by resorting to an online calculator (www.mccallum-layton.co.uk/tools/statistic-calculators/sam ple-size-calculator). Importantly, tumor samples are characterized by a high degree of cellular heterogeneity. Although the analysis of 380 cells is highly feasible with the imaging framework in any pathology laboratory, it is important to emphasize that more than assessing a discrete a number of cells, it is important to assess a robust sampling coverage of the region of interest pre-defined by the pathologist. This is instrumental for the representativeness and, thus clinical relevance of the analysis of a tumor sample. This new imaging method is a highly sensitive classification tool as it generates very low false negative rates, that is, it misses very few true DAPI-stained nuclei classifications. Regardless its potential screening value, the analytical and predictive value of this imaging framework in clinical analysis is dependent on the coverage of the samples collected (representativeness), which reflects on the overall time of analysis. Depending on the objective amplification used, image acquisitions will yield different number of cells per field-of-view, meaning that a variable number of images will be needed to reach a representative estimation of the whole sample and this will, ultimately, impact on the time of analysis. Hence, its application to molecular pathology, such as in cancer, will be highly dependent of these factors but will allow, in an operator semi-dependent manner, a more objective and quantitative approach to molecular pathology, reducing the operator bias and the analysis subjectivity. In this sense, the application of this imaging framework to clinical settings should be envisage, globally, as a complementary approach to corroborate, or challenge, molecular pathology.

Supplementary Information accompanies the paper on the Laboratory Investigation website (http://www.laboratoryinvestigation.org)

\section{ACKNOWLEDGMENTS}

This work was financed by FEDER-Fundo Europeu de Desenvolvimento Regional funds through the COMPETE 2020-Operacional Programme for Competitiveness and Internationalization (POCI), Portugal 2020, and by Portuguese funds through FCT - Fundação para a Ciência e a Tecnologia/Ministério da Ciência, Tecnologia e Inovação in the framework of the project 'Institute for Research and Innovation 
in Health Sciences' (POCl-01-0145-FEDER-007274), PTDC/BIM-ONC/0171/2012, PTDC/BIM-ONC/0281/2014 and PTDC/BBB-IMG/0283/2014. AF is an FCT post-doctoral fellowship recipient (SFRH/BPD/97295/2013) and PC is funded by NORTE-01-0145-FEDER-000029. We gratefully acknowledge Patrícia Oliveira and Joana Carvalho for the critical reading of the manuscript and insightful discussion, Cecília Durães for her assistance in statistical analyses and João Vinagre for initial assistance in image acquisition. Imaging art was created using Servier Medical Art (www.servier.com).

\section{DISCLOSURE/CONFLICT OF INTEREST}

The authors declare no conflict of interest.

1. Vermeulen K, Van Bockstaele DR, Berneman ZN. The cell cycle: a review of regulation, deregulation and therapeutic targets in cancer. Cell Prolif 2003;36:131-149.

2. Darzynkiewicz Z. Critical aspects in analysis of cellular DNA content. Curr Protoc Cytom 2011;Chapter 7, Unit 72: 7.2.1-7.2.8.

3. Henderson L, Bortone DS, Lim C, et al. Classic "broken cell" techniques and newer live cell methods for cell cycle assessment. Am J Physiol Cell Physiol 2013;304:C927-C938.

4. Malumbres M, Barbacid M. Cell cycle, CDKs and cancer: a changing paradigm. Nat Rev Cancer 2009;9:153-166.

5. Danielsen HE, Pradhan M, Novelli M. Revisiting tumour aneuploidy-the place of ploidy assessment in the molecular era. Nat Rev Clin Oncol 2016;13:291-304.

6. Sarah P, David M. Mechanisms of anticancer drugs. In: Michael G (ed). Scott-Brown's Otorhinolaryngology: Head and Neck Surgery, 7 edn. CRC Press: Hodder Arnold, London, 2008, pp 34-46.

7. Seiwert TY, Salama JK, Vokes EE. The concurrent chemoradiation paradigm-general principles. Nat Clin Pract Oncol 2007;4:86-100.

8. Pozarowski P, Holden E, Darzynkiewicz Z. Laser scanning cytometry: principles and applications-an update. Methods Mol Biol 2013;931:187-212.

9. Chattopadhyay PK, Roederer M. A mine is a terrible thing to waste: high content, single cell technologies for comprehensive immune analysis. Am J Transplant 2015;15:1155-1161.

10. Sakaue-Sawano A, Miyawaki A. Visualizing spatiotemporal dynamics of multicellular cell-cycle progressions with fucci technology. Cold Spring Harb Protoc 2014;2014:525-531.

11. Sakaue-Sawano A, Kobayashi T, Ohtawa K, et al. Drug-induced cell cycle modulation leading to cell-cycle arrest, nuclear mis-segregation, or endoreplication. BMC Cell Biol 2011;12:2.

12. Rasband WS. ImageJ. Available at http://imagej.nih.gov/ij/U. S. National Institutes of Health: Bethesda, MD, USA, 1997-2015.

13. Mestre T, Figueiredo J, Ribeiro AS, et al. Quantification of topological features in cell meshes to explore E-cadherin dysfunction. Sci Rep 2016;6:25101.

14. Gonzalez RC, Woods RE, Eddins SL. Digital Image Processing Using MATLAB, Second edn. Gatesmark Publishing: Knoxville, 2009.

15. Kapuscinski J. DAPI: a DNA-specific fluorescent probe. Biotech Histochem 1995;70:220-233.

16. Bishop C. Pattern Recognition and Machine Learning. Springer-Verlag New York, NY, USA. 2006.

17. Moon TK, Stirling WC. Mathematical Methods and Algorithms for Signal Processing. Prentice-Hall, Inc.: Upper Saddle River, NJ, USA. 2000.
18. Hess AS, Shardell M, Johnson JK, et al. Methods and recommendations for evaluating and reporting a new diagnostic test. Eur J Clin Microbiol Infect Dis 2012;31:2111-2116.

19. Greenland S, Senn SJ, Rothman KJ, et al. Statistical tests, P values, confidence intervals, and power: a guide to misinterpretations. Eur Epidemiol 2016;31:337-350.

20. Parikh $\mathrm{R}$, Mathai $\mathrm{A}$, Parikh $\mathrm{S}$, et al. Understanding and using sensitivity, specificity and predictive values. Indian J Ophthalmol 2008;56:45-50.

21. Sakaue-Sawano A, Kurokawa $H$, Morimura $T$, et al. Visualizing spatiotemporal dynamics of multicellular cell-cycle progression. Cell 2008;132:487-498.

22. Altman DG, Bland JM. Diagnostic tests. 1: sensitivity and specificity. BMJ 1994;308:1552.

23. Maxim LD, Niebo R, Utell MJ. Screening tests: a review with examples. Inhal Toxicol 2014;26:811-828.

24. Grimes DA, Schulz KF. Refining clinical diagnosis with likelihood ratios. Lancet 2005;365:1500-1505.

25. Sanches JM, Figueiredo J, Fonseca $M$, et al. Quantification of mutant E-cadherin using bioimaging analysis of in situ fluorescence microscopy. A new approach to $\mathrm{CDH} 1$ missense variants. Eur J Hum Genet 2015;23:1072-1079.

26. Rosenblatt J. Mitosis: moesin and the importance of being round. Curr Biol 2008; 18:R292-R293.

27. Roukos V, Pegoraro G, Voss TC, et al. Cell cycle staging of individual cells by fluorescence microscopy. Nat Protoc 2015;10:334-348.

28. Wegner KD, Hildebrandt N. Quantum dots: bright and versatile in vitro and in vivo fluorescence imaging biosensors. Chem Soc Rev 2015;44: 4792-4834.

29. Bruhn C, Kroll T, Wang ZQ. Systematic characterization of cell cycle phase-dependent protein dynamics and pathway activities by high-content microscopy-assisted cell cycle phenotyping. Genomics Proteomics Bioinform 2014;12:255-265.

30. Zielke N, Edgar BA. FUCCI sensors: powerful new tools for analysis of cell proliferation. Wiley Interdiscip Rev Dev Biol 2015:4:469-487.

31. Mort RL, Ford MJ, Sakaue-Sawano A, et al. Fucci2a: a bicistronic cell cycle reporter that allows Cre mediated tissue specific expression in mice. Cell Cycle 2014;13:2681-2696.

32. Zielke N, Korzelius J, van Straaten M, et al. Fly-FUCCl: a versatile tool for studying cell proliferation in complex tissues. Cell Rep 2014;7:588-598.

33. Bertero A, Vallier L. Fucci2a mouse upgrades live cell cycle imaging. Cell Cycle 2015;14:948-949.

34. Padfield D, Rittscher J, Thomas $\mathrm{N}$, et al. Spatio-temporal cell cycle phase analysis using level sets and fast marching methods. Med Image Anal 2009;13:143-155.

35. Camp RL, Neumeister V, Rimm DL. A decade of tissue microarrays: progress in the discovery and validation of cancer biomarkers. J Clin Oncol 2008;26:5630-5637.

36. Yaziji H, Barry T. Diagnostic Immunohistochemistry: what can go wrong? Adv Anat Pathol 2006:13:238-246.

37. Marcos R, Monteiro RA, Rocha E. The use of design-based stereology to evaluate volumes and numbers in the liver: a review with practical guidelines. J Anat 2012;220:303-317.

38. Lahrmann B, Halama N, Sinn HP, et al. Automatic tumor-stroma separation in fluorescence TMAs enables the quantitative highthroughput analysis of multiple cancer biomarkers. PLoS ONE 2011;6: e28048. 